Volume 7

Issue 4 -- Women and Cancer

Article 5

$10-23-2020$

\title{
Validation of an Endometrial Tumor Diameter Model for Risk Assessment in the Absence of Lymph Node Mapping
}

McKayla J. Riggs

Callie M. Cox Bauer

Caela R. Miller

James K. Aden

Scott A. Kamelle

Follow this and additional works at: https://aah.org/jpcrr

Part of the Female Urogenital Diseases and Pregnancy Complications Commons, Neoplasms

Commons, Obstetrics and Gynecology Commons, Oncology Commons, Surgery Commons, Surgical

Procedures, Operative Commons, and the Urogenital System Commons

\section{Recommended Citation}

Riggs MJ, Cox Bauer CM, Miller CR, Aden JK, Kamelle SA. Validation of an endometrial tumor diameter model for risk assessment in the absence of lymph node mapping. J Patient Cent Res Rev. 2020;7:323-8. doi: 10.17294/2330-0698.1768

Published quarterly by Midwest-based health system Advocate Aurora Health and indexed in PubMed Central, the Journal of Patient-Centered Research and Reviews (JPCRR) is an open access, peer-reviewed medical journal focused on disseminating scholarly works devoted to improving patient-centered care practices, health outcomes, and the patient experience. 


\title{
Validation of an Endometrial Tumor Diameter Model for Risk Assessment in the Absence of Lymph Node Mapping
}

\author{
McKayla J. Riggs, MD, ${ }^{1}$ Callie M. Cox Bauer, DO, ${ }^{1,2}$ Caela R. Miller, MD, ${ }^{1}$ James K. Aden, PhD, ${ }^{3}$ \\ Scott A. Kamelle, MD ${ }^{4}$ \\ ${ }^{1}$ Department of Obstetrics and Gynecology, Brooke Army Medical Center, San Antonio, TX; ${ }^{2}$ Obstetrics and \\ Gynecology, Aurora Sinai Medical Center, Milwaukee, WI; ${ }^{3}$ Brooke Army Medical Center, San Antonio, TX; \\ ${ }^{4}$ Gynecologic Oncology, Aurora St. Luke's Medical Center, Milwaukee, WI
}

Purpose

This study aimed to assess the optimal tumor diameter for predicting lymphatic metastasis and to determine intraoperatively the need for lymph node dissection in patients with endometrioid endometrial cancer.

Methods

Military beneficiaries diagnosed with stage I-III endometrioid endometrial cancer during 2003-2016 who had at least 7 pelvic and/or paraaortic lymph nodes removed during the time of hysterectomy were studied. Tumor diameter was compared against the presence of positive nodes, using the prior models of $20 \mathrm{~mm}$ (ie, Mayo model) and $50 \mathrm{~mm}$ (ie, Milwaukee model), to determine the false-negative rate of each threshold. A separate analysis was completed to determine the optimal diameter for our population. Receiver operating characteristic curve analysis models of tumor diameter were evaluated for model fit and predictive power of lymph node involvement.

Results Of the 1224 patients with endometrioid endometrial cancer included, 13\% ( $\mathrm{n}=160)$ had positive lymph node involvement. Tumor sizes ranged from $1 \mathrm{~mm}$ to $100 \mathrm{~mm}$. In contrast to Mayo and Milwaukee models (ie, Mayo, Milwaukee), the optimal tumor diameter independent of myometrial invasion and grade of tumor to predict lymph node metastasis was found to be $35 \mathrm{~mm}$.

Conclusions Endometrioid endometrial cancer tumor diameter of $35 \mathrm{~mm}$ was found to be the optimal threshold for lymphadenectomy when the operating surgeon has no knowledge of tumor invasion. ( $J$ Patient Cent Res Rev. 2020;7:323-328.)

Keywords endometrioid endometrial cancer; lymph node involvement; tumor diameter; lymphadenectomy

$\mathrm{U}$ terine cancer is the fourth most common form of female cancer in the United States and remains the most commonly diagnosed gynecologic cancer, accounting for 57,368 diagnoses in 2017 and 10,994 deaths. ${ }^{1}$ According to the National Institutes of Health's Surveillance, Epidemiology, and End Results Program database, 65,620 new cases of uterine cancer are expected by the conclusion of 2020, affecting 27.8 per 100,000 women and comprising $3.6 \%$ of all new cancer case diagnoses. Current lifetime risk is

Corresponding author: Callie M. Cox Bauer, DO, Aurora Sinai Medical Center OB/GYN Residency, 945 N. 12th Street, Milwaukee, WI 53223

(callie.coxbauer@aah.org) estimated to be $3.1 \% .^{2}$ In 2017 , there were an estimated 793,846 women living with uterine cancer in the United States. Worldwide, uterine cancer continues to be the most common gynecologic cancer and the eighth most common cancer. ${ }^{3}$

Due to its prevalence, appropriate surgical management is crucial for optimal prognosis. Currently, the standard of care includes total hysterectomy and bilateral salpingooophorectomy with complete lymphadenectomy for all patients at risk of lymphatic dissemination. ${ }^{4,5}$ Since 1988, the International Federation of Obstetrics and Gynecology (FIGO) has recommended surgical staging for endometrial cancer patients, as lymph node involvement is an important prognostic indicator. ${ }^{6,7}$ Complete lymphadenectomy, however, has significant morbidity and no change in mortality rates for low- 
risk patients. ${ }^{5,7-11}$ There is also considerable variation in type of lymphadenectomy that can be performed. The extent of lymph node assessment for what is considered optimal patient management has varied from sentinel node mapping to complete pelvic and paraaortic lymphadenectomy.

Because of this variation, disease management and risk scoring models were developed to personalize intraoperative or postoperative care by predicting the probability of a clinical event or outcome. ${ }^{12,13}$ Utilizing these models to identify low-risk patients and avoid lymphadenectomy to decrease morbidity was the goal. The definition of a low-risk patient varies, with the currently used risk scoring models including tumor variables that are not always readily available intraoperatively. Hence, these models have had little significance on standardizing intraoperative surgical management.

The use of sentinel lymph node mapping by gynecologic oncologists is increasingly accepted in community practice for low- to intermediate-risk patients, but details about its implementation and what to do with high-risk patients remain controversial. ${ }^{14-16}$ Use of sentinel nodes as a solution is limited by the technical reality that the injections for mapping must be performed prior to uterine removal. If a previously undiagnosed tumor is discovered intraoperatively after uterine removal, other strategies must be used to determine lymph node status in low- and intermediate-risk patients. Furthermore, endometrial cancer surgeries may or may not be performed by gynecologic oncologists. ${ }^{17}$ Given these realities of undiagnosed cancer and possible sentinel lymph node mapping failure, there will always be a need for intraoperative risk assessment that relies on easily obtained tumor-related information.

The most commonly used criteria for predicting individualized risk of lymphatic dissemination in patients with endometrioid endometrial cancer (EEC), a type of uterine cancer, is based on a study by Mayo Clinic (Rochester, MN). ${ }^{10}$ The Mayo model, first published in 2000, includes presence of lymphovascular space invasion (LVSI), myometrial invasion of $>50 \%$, FIGO grade I or II, primary tumor diameter of $\geq 2 \mathrm{~cm}$ (ie, $\geq 20$ $\mathrm{mm}$ ), and cervical stromal invasion. In the Mayo model, a tumor diameter greater than $20 \mathrm{~mm}$ confers a 5-fold increased risk of lymphatic dissemination. Subsequently, elimination of tumor diameter from risk stratification resulted in an overestimation of the risk of lymphatic dissemination. ${ }^{13}$ However, since tumor diameter has not been an independent predictor of lymph node metastasis, national and international guidelines for management of EEC have not emphasized its usefulness for intraoperative decisions. ${ }^{18}$ Regardless of retrospective analysis of tumor-related risk factors for lymph node metastasis, tumor diameter remains an easily collected piece of intraoperative information available to the surgeon, whereas LVSI status, grade, and sometimes even myometrial invasion are not immediately available. Sentinel lymph node dye injection must be performed prior to uterine removal and before many of the tumorrelated risk factors are known.

A retrospective study encompassing data from the 15-hospital Aurora Health Care health system (Milwaukee, WI) on 727 patients with cancer was performed by Cox Bauer et al and published in 2016. ${ }^{13}$ Their study confirmed tumor diameter was an important predictor of lymphatic metastasis in patients diagnosed with early-stage endometrial cancer and indicated that predictability of lymphatic dissemination might be improved by increasing the tumor diameter cutoff from the previously proposed Mayo model of $20 \mathrm{~mm}$ to $50 \mathrm{~mm}$ and lowering the myometrial invasion cutoff from $50 \%$ to $33 \%$ (ie, the Milwaukee model). The data revealed a $3.1 \%$ increase in odds of lymphatic dissemination per 1-mm increase in tumor diameter, a $3.7 \%$ increase in lymphatic dissemination per $1 \%$ increase in myometrial invasion, and an increase in overall sensitivity on determining risk of lymphatic dissemination using the new criteria. These results suggested that tumor diameter can function as an alternative predictor of lymphatic metastasis at hospitals that do not have pathology readily available to evaluate myometrial invasion or when the intraoperative analysis is unclear.

Several studies have demonstrated the significance of tumor diameter as an important prognostic indicator, though most use the threshold value of $20 \mathrm{~mm}$ from the NCCN guidelines. ${ }^{14,19}$ Our goal for the study presented herein is to examine if tumor diameter alone can be utilized to predict lymph node dissemination and evaluate if there is an optimal tumor size for predicting lymphatic spread. We also aim to provide external validity of the Milwaukee model by evaluating tumor diameter and lymph node metastasis in the Military Health System (Falls Church, VA), which provides a larger, more geographically diverse, variable patient population. Determining whether the optimal tumor size of $20 \mathrm{~mm}$ versus $50 \mathrm{~mm}$ for low-risk patients with EEC in this population is consistent with prior models is the primary goal, with the secondary objective of exploring whether there is a more optimal tumor diameter to best predict lymphatic involvement.

\section{METHODS}

A retrospective analysis of adult patients diagnosed with EEC was completed. Patients were 18 years of age and older, seen within the U.S. Department of Defense 
Military Health System from January 2003 to May 2016, and registered with the Automated Central Tumor Registry (ACTUR). The use of pelvic and/or paraaortic lymphadenectomy during hysterectomy was the standard for all EEC cases during this period. Patient inclusion was based on final pathologic diagnosis of endometrioid histology; patients with less than 7 lymph nodes removed, nonendometrioid histology, stage IV classification, and/ or missing information regarding lymph node status were excluded from the study. Patient demographics as well as tumor and treatment characteristics were identified from the U.S. Department of Defense cancer registry. The Brooke Army Medical Center (San Antonio, TX) institutional review board approved the study (\#C.2017.092n).

Categorical data were summarized by percentages and analyzed using the chi-squared test or Fisher's exact test, whichever was most appropriate. Means and standard deviations (SD) or medians were used as summary statistics for continuous variables. Logistic regression was performed to determine the predictive qualities of various factors associated with positive nodes. Unlike the Mayo and Milwaukee models, this model only uses tumor diameter and does not include information about LVSI or myometrial invasion. Odds ratios, along with their corresponding Wald's 95\% confidence intervals, were reported.

Receiver operating characteristic (ROC) analysis also was performed to determine the predictive quality of the model. Finally, ROC curves were used to determine which cutoffs optimized sensitivity and specificity of predicting a positive node. Significance for results was established when P-values were less than 0.05. All statistical analysis was performed using SPSS Statistics V22.0 software (IBM Corporation).

\section{RESULTS}

A total of 1300 patients diagnosed with EEC via surgical pathology were found in the ACTUR database from
2003 to 2016. After patients without at least 7 lymph nodes (61 patients) and stage IV patients were removed, a total of 1224 patients remained. Of those 1224,160 patients had positive lymph node status (13.1\%), 800 patients had a tumor size of $\geq 20 \mathrm{~mm}$ in size $(65.4 \%)$, and 253 had a tumor size of $\geq 50 \mathrm{~mm}(20.7 \%)$. Patients ranged in age from 18 to 90 years, with the majority in the 50-65-year-old bracket. The mean age was 54 years (SD: 13 years); $67 \%$ of the population was White nonHispanic, 9\% was African American, 5\% was Filipino, $2 \%$ was Pacific Islander, and 1\% was Japanese, Korean, or Micronesian.

The mean tumor size was $39 \mathrm{~mm}$ (SD: $78 \mathrm{~mm}$ ). The presence of LVSI was reported in 588 (48\%) of the 1224 patients with EEC analyzed, with $132(22 \%)$ of those 588 patients found LVSI-positive. Unlike the Mayo and Milwaukee models, this model only used tumor diameter and did not include information about myometrial invasion or tumor grade.

Individual ROC curves were analyzed to determine the best fit, both as a continuous variable and by using the $20-\mathrm{mm}$ and $50-\mathrm{mm}$ tumor diameter cutoffs from the Mayo and Milwaukee models (Table 1; Figures 1-3). Sensitivity and specificity at $20 \mathrm{~mm}$ were $81.9 \%$ and $37.1 \%$, respectively. Sensitivity and specificity at $50 \mathrm{~mm}$ were $82.1 \%$ and $39.4 \%$, respectively (Table 2). Positive predictive value at $20 \mathrm{~mm}$ was $16.4 \%$ and at $50 \mathrm{~mm}$ was $24.9 \%$. Negative predictive value at $20 \mathrm{~mm}$ was $92.9 \%$ and at $50 \mathrm{~mm}$ was $90.0 \%$. The false-negative rate was $6.8 \%$ for $20 \mathrm{~mm}$ and $10.0 \%$ for $50 \mathrm{~mm}$.

The continuous ROC curve optimized sensitivity and specificity with an ideal tumor size of $35 \mathrm{~mm}$ for this patient population, which falls between the two values proposed by the Mayo and Milwaukee models. A tumor diameter cutoff of $35 \mathrm{~mm}$ resulted in sensitivity of $65.6 \%$ and specificity of $63.0 \%$, positive and negative predictive values of $21.0 \%$ and $92.4 \%$, respectively, and a falsenegative rate of $7.6 \%$ (Table 2 ).

Table 1. Comparison of Risk Assessment Models Using Tumor Diameter Only*

\begin{tabular}{l|l|l|l|l}
\hline Model & $\boldsymbol{R}^{\mathbf{2}}(\mathbf{U})$ & AICc & BIC & AUC \\
\hline Tumor diameter (continuous) & 0.0046 & 948.9 & 959.1 & 0.671 \\
Tumor diameter $\geq 20 \mathrm{~mm}$ & 0.0256 & 928.9 & 939.1 & 0.595 \\
Tumor diameter $\geq 50 \mathrm{~mm}$ & 0.0362 & 918.9 & 929.1 & 0.608 \\
\hline
\end{tabular}

*Evaluation of the 20-mm and 50-mm cutoffs in our patient population confirmed that the 50-mm model performed better.

$A I C c$, Akaike information criterion with correction; AUC, area under the curve; BIC, Bayesian information criterion. 


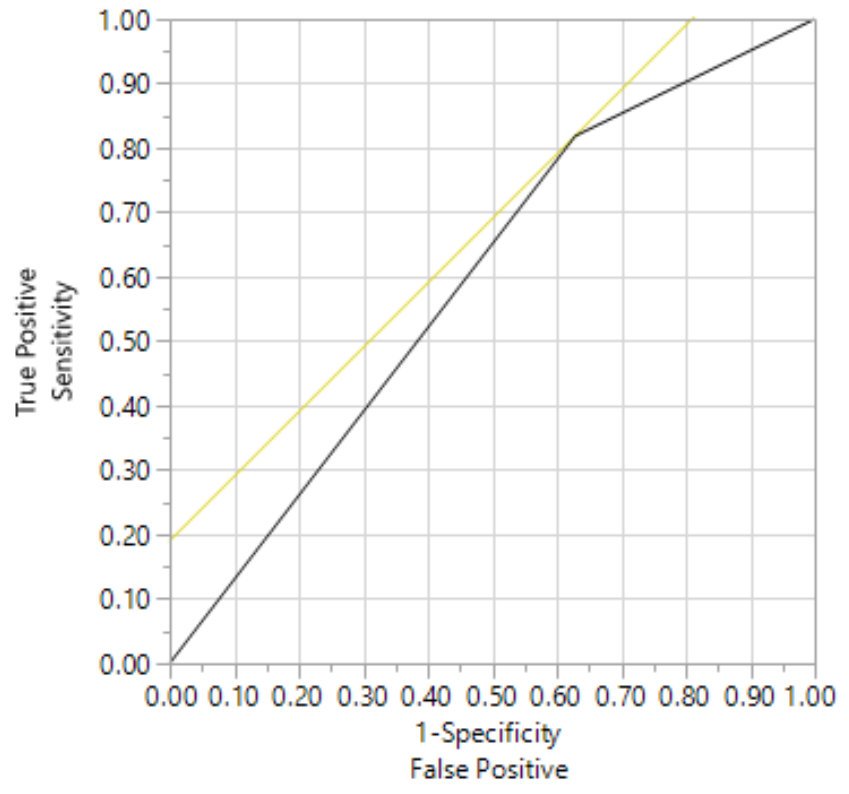

Figure 1. Lymphatic dissemination models containing tumor diameter only were analyzed using receiver operating characteristic (ROC) curves. Shown here is the ROC curve (black) and iso-performance line (yellow) for a model containing 20-mm tumor diameter only.

\section{DISCUSSION}

Recently, Cox Bauer et al proposed the Milwaukee risk model as an alternative to the long-used Mayo criteria for patients with EEC, citing the former's increased sensitivity and specificity in predicting lymph nodepositive status with cutoffs for tumor diameter of $\geq 50$ $\mathrm{mm}$ and myometrial invasion of $>33 \% .{ }^{13}$ That study highlighted the importance of tumor diameter in risk assessment, even more so than the extent of myometrial invasion, once tumor diameter was examined as both continuous and categorical variables. Our study sought to determine external validity of the Milwaukee model and analyze tumor diameter as a continuous variable in a nationwide military population. In Milwaukee model data, there was a $3.1 \%$ increased odds of lymph node metastasis for every 1-mm increase in tumor diameter. This was consistent with the collected LVSI and depth of invasion risk of lymph node metastasis data collected in our study population.

As myometrial invasion was not consistently available throughout the ACTUR database, the main focus of our study became tumor diameter and whether the Milwaukee model could be recreated solely using tumor diameter as a continuous variable. The ACTUR data validated the Milwaukee model's suggestion that, compared to $20 \mathrm{~mm}$, $50 \mathrm{~mm}$ serves as a better cutoff threshold, with a superior positive predictive value $(24.9 \%$ vs $16.4 \%)$ and a small effect on the false-negative rate $(10.0 \%$ for $50 \mathrm{~mm}$ vs

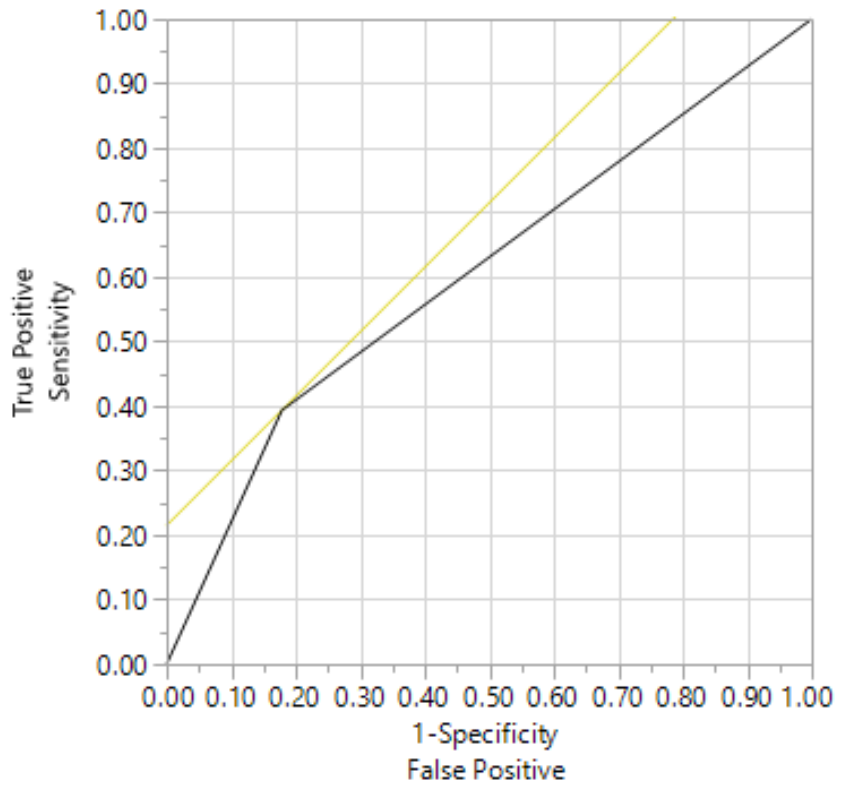

Figure 2. Receiver operating characteristic curve for a lymphatic dissemination model containing $50-\mathrm{mm}$ tumor diameter only.

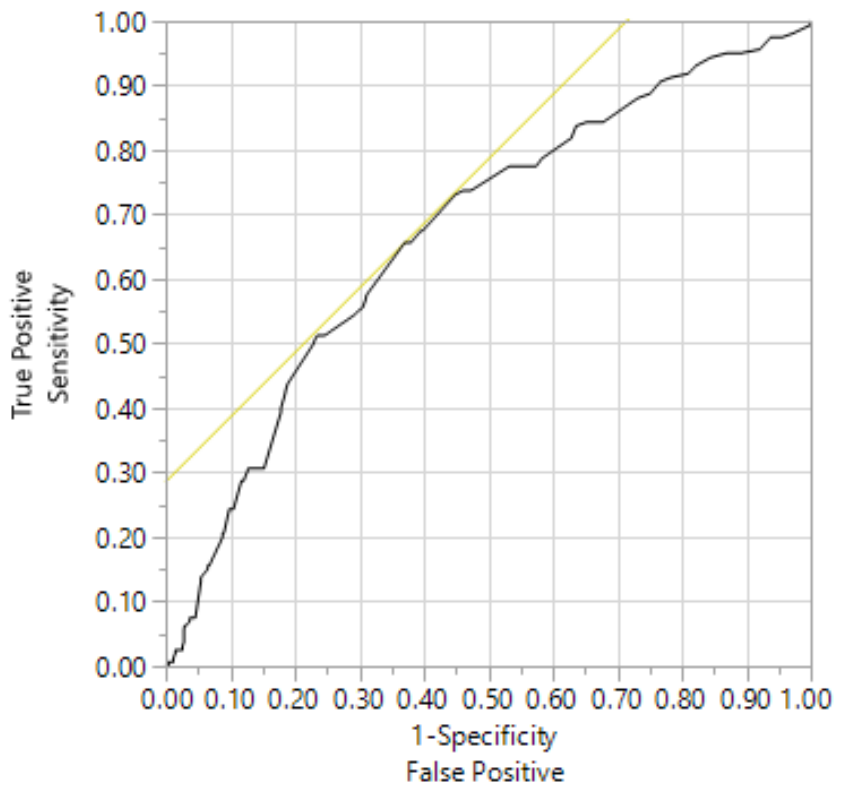

Figure 3. Receiver operating characteristic curve for continuous tumor diameter, which revealed a 35- $\mathrm{mm}$ cutoff as having the most optimal sensitivity and specificity to predict risk of lymph node involvement.

$6.8 \%$ for $20 \mathrm{~mm}$ ). Of further interest, when ACTUR data were used to analyze the optimal tumor diameter to maximize sensitivity and specificity, $35 \mathrm{~mm}$ was noted to be the optimal threshold, with positive $(21.0 \%)$ and negative $(92.4 \%)$ predictive values falling between the two previous models and a false-negative rate of $7.6 \%$. 
Table 2. Sensitivity and Specificity of Three Tumor Diameter Models

\begin{tabular}{lcc}
\hline Tumor diameter & Specificity & Sensitivity \\
\hline $35 \mathrm{~mm}$ & 0.6297 & 0.6563 \\
$20 \mathrm{~mm}$ & 0.3712 & 0.8188 \\
$50 \mathrm{~mm}$ & 0.8214 & 0.3938 \\
\hline
\end{tabular}

Ideal risk stratification for spread of EEC requires the presence of three key pieces of information: LVSI, percentage of myometrial invasion, and tumor diameter. However, sometimes tumor diameter is the only piece of information available to the surgeon. We propose that a tumor diameter of $35 \mathrm{~mm}$, rather than the $20 \mathrm{~mm}$ used in the Mayo model or the $50 \mathrm{~mm}$ suggested by the Milwaukee model, should serve as the cutoff value for this risk factor. By increasing the level of tumor size from Mayo's criteria, our proposed model would prevent an additional number of unnecessary lymphadenectomies. Importantly, this less comprehensive model would be intended for intraoperative use when a patient's sentinel lymph nodes cannot be identified, when a tumor was not recognized prior to surgery, or when frozen pathology is inconclusive or not available. While we hope that these situations are rare, it is our opinion this tumor diameter model can be utilized to achieve reasonable outcomes.

EEC is one of the most common female cancers, and our model becomes valuable when gross evidence of tumor is found in patients during the aforedescribed situations. Tumor qualities that are most predictive of lymph node dissection - LVSI, grade, and depth of invasion are not readily available at all times in all institutions, nor in patients for whom attempted identification of a sentinel lymph node fails. In rural or community settings with limited pathology support, we suggest that tumor diameter alone is a potent predictor of lymphatic metastasis, thereby allowing an easy method to determine the extent of lymph node dissection needed.

As sentinel lymph node dissection becomes more routine, tumor diameter models may become less important. However, sentinel lymph node mapping failures do occur, even at centers where they are commonly performed. Depending on technique, identification of bilateral lymph nodes is reported to range from $66 \%$ to $97 \% .^{20,21}$ At this time, National Comprehensive Cancer Network guidelines recommend a full lymph node dissection should sentinel node mapping fail. ${ }^{16}$ A scoring system based on tumor size may help avoid morbidity in this subset of patients. Furthermore, although patients with endometrial intraepithelial neoplasia (EIN) have as high as a $43 \%$ risk of concurrent endometrial cancer, there is lack of consensus if sentinel lymph node mapping or frozen section should be performed in patients with EIN. ${ }^{22}$ Thus, for these patients, examination of the uterus for tumor and measurement of tumor diameter could be used to predict the risk of lymphatic spread and need for lymphadenectomy. Our model can be used to determine how to proceed in patients who have unpredicted gross tumor on intraoperative uterine inspection.

\section{Limitations}

Limitations of the study include absence of myometrial invasion and LVSI information for a large subset of the patient cohort. Data were reported to a database and then retrieved for this study; as such, missing data from multiple records was noted. This approach limited our ability to reconstruct the Mayo and Milwaukee models completely using our dataset.

\section{CONCLUSIONS}

Patients with endometrioid endometrial cancer registered in the Military Health System cancer database (ACTUR) were used as a validation population for the Milwaukee model of metastatic risk assessment, which uses tumor diameter to predict presence of lymph node metastasis and suggests that tumor diameter alone can be used to predict lymph node involvement of disease. Our study validated the Milwaukee model's 50-mm threshold for tumor diameter but also found that, when relying on tumor size only (a clinically relevant scenario), forgoing lymph node dissection in endometrioid endometrial tumors less than $35 \mathrm{~mm}$ in diameter resulted in an acceptable false-negative rate.

\section{Patient-Friendly Recap}

- Assessing risk in patients with uterine cancer based on tumor size would allow clinicians to determine whether or not a lymphadenectomy is needed without taking a frozen section or relying on sentinel lymph node identification.

- The authors analyzed a large, national patient population to validate if tumor size alone can adequately assess patient risk, especially in clinical scenarios where lymph node mapping fails or is unavailable.

- In addition to verifying tumor diameter as a reliable standalone risk factor, they identified $35 \mathrm{~mm}$ as the optimal cutoff for sufficiently assessing patient risk while avoiding surgery.

- Further validation of this $35-\mathrm{mm}$ tumor diameter threshold is needed. 


\section{Author Contributions}

Study design: Cox Bauer, Miller, Kamelle. Data acquisition or analysis: Riggs, Cox Bauer, Miller, Aden. Manuscript drafting: Riggs, Cox Bauer. Critical revision: Cox Bauer, Miller, Kamelle.

\section{Conflicts of Interest}

The authors have no conflicts to disclose. The views expressed herein are those of the authors and do not reflect the official policy or position of Brooke Army Medical Center, the U.S. Army Medical Department, the U.S. Army Office of the Surgeon General, the Department of the Army, the Department of the Air Force, the Department of Defense, or the U.S. Government.

\section{References}

1. Centers for Disease Control and Prevention; U.S. Cancer Statistics. Leading cancer cases and deaths, all races/ethnicities, male and female, 2017. Page based on 2019 submission data; accessed July 12, 2020. www.cdc.gov/cancer/dataviz

2. National Cancer Institute. Cancer stat facts: uterine cancer. Accessed July 12, 2020. https://seer.cancer.gov/statfacts/html/ corp.html

3. Cancer Today. Cancer fact sheets: corpus uteri. Published 2018; accessed July 12, 2020. https://gco.iarc.fr/today/factsheets-cancers

4. Frost JA, Webster KE, Bryant A, Morrison J. Lymphadenectomy for the management of endometrial cancer. Cochrane Database Syst Rev. 2015;(9):CD007585. Crossref

5. ASTEC study group; Kitchener H, Swart AM, Qian Q, Amos C, Parmar MK. Efficacy of systematic pelvic lymphadenectomy in endometrial cancer (MRC ASTEC trial): a randomised study. Lancet. 2009;373:125-36. Crossref

6. Dowdy SC, Glaser GE, Lurain JR. Uterine cancer. In: Berek JS (ed). Berek \& Novak's Gynecology, Sixteenth Edition. Wolters Kluwer; 2020, pp. 1002-37.

7. Creasman WT, Morrow CP, Bundy BN, Homesley HD, Graham JE, Heller PB. Surgical pathologic spread patterns of endometrial cancer. A Gynecologic Oncology Group Study. Cancer. 1987;60(8 Suppl):2035-41. 당ssref

8. Creasman WT, Odicino F, Maisonneuve P, et al. Carcinoma of the corpus uteri. FIGO 26th Annual Report on the Results of Treatment in Gynecological Cancer. Int J Gynaecol Obstet. 2006;95 Suppl 1:S105-43. Crossref

9. Benedetti Panici P, Basile S, Maneschi F, et al. Systematic pelvic lymphadenectomy vs. no lymphadenectomy in earlystage endometrial carcinoma: randomized clinical trial. $J$ Natl Cancer Inst. 2008;100:1707-16. Crossref

10. Mariani A, Webb MJ, Keeney GL, Haddock MG, Calori G, Podratz KC. Low-risk corpus cancer: Is lymphadenectomy or radiotherapy necessary? Am J Obstet Gynecol. 2000;182:1506-19. $\underline{\text { Crossref }}$
11. Dowdy SC, Borah BJ, Bakkum-Gamez JN, et al. Prospective assessment of survival, morbidity, and cost associated with lymphadenectomy in low-risk endometrial cancer. Gynecol Oncol. 2012;127:5-10. Crossref

12. Milam MR, Java J, Walker JL, et al. Nodal metastasis risk in endometrioid endometrial cancer. Obstet Gynecol. 2012;119 (2 Pt 1):286-92. Crossref

13. Cox Bauer CM, Greer DM, Kram JJ, Kamelle SA. Tumor diameter as a predictor of lymphatic dissemination in endometrioid endometrial cancer. Gynecol Oncol. 2016;141:199-205. Crossref

14. Koh WJ, Abu-Rustum NR, Bean S, et al. Uterine neoplasms, version 1.2018, NCCN Clinical Practice Guidelines in Oncology. J Natl Compr Canc Netw. 2018;16:170-99. Crossref

15. Rossi EC, Kowalski LD, Scalici J, et al. A comparison of sentinel lymph node biopsy to lymphadenectomy for endometrial cancer staging (FIRES trial): a multicentre, prospective, cohort study. Lancet Oncol. 2017;18:384-92. Crossref

16. Chambers LM, Vargas R, Michener CM. Sentinel lymph node mapping in endometrial and cervical cancer: a survey of practices and attitudes in gynecologic oncologists. J Gynecol Oncol. 2019;30(3):e35. Crossref

17. Ramzan AA, Behbakht K, Corr BR, Sheeder J, Guntupalli SR. Minority race predicts treatment by non-gynecologic oncologists in women with gynecologic cancer. Ann Surg Oncol. 2018;25:3685-91. Crossref

18. Vargas R, Rauh-Hain JA, Clemmer J, et al. Tumor size, depth of invasion, and histologic grade as prognostic factors of lymph node involvement in endometrial cancer: a SEER analysis. Gynecol Oncol. 2014;133:216-20. Crossref

19. Holloway RW, Molero Bravo RA, Rakowski JA, et al. Detection of sentinel lymph nodes in patients with endometrial cancer undergoing robotic-assisted staging: a comparison of colorimetric and fluorescence imaging. Gynecol Oncol. 2012;126:25-9. Crossref

20. Niikura H, Kaiho-Sakuma M, Tokunaga H, et al. Tracer injection sites and combinations for sentinel lymph node detection in patients with endometrial cancer. Gynecol Oncol. 2013;131:299-303. Crossref

21. Daraï E, Dubernard G, Bats AS, et al. Sentinel node biopsy for the management of early stage endometrial cancer: longterm results of the SENTI-ENDO study. Gynecol Oncol. 2015;136:54-9. Crossref

22. Trimble CL, Kauderer J, Zaino R, et al. Concurrent endometrial carcinoma in women with a biopsy diagnosis of atypical endometrial hyperplasia: a Gynecologic Oncology Group study. Cancer. 2006;106:812-9. Crossref

(C) 2020 Advocate Aurora Health, Inc. 